\title{
Congenital choanal atresia: our experiences in a tertiary care teaching hospital in eastern India
}

\author{
Santosh Kumar Swain ${ }^{1}$, Mahesh Chandra Sahu², Asirbad Jena' \\ 'Department of Otorhinolaryngology, IMS and SUM Hospital, Siksha "O" Anusandhan University, K8, Kalinganagar, \\ Bhubaneswar-751003, Odisha, India \\ 2Directorate of Medical Research, IMS and SUM Hospital, Siksha "O" Anusandhan University, K8, Kalinganagar, \\ Bhubaneswar-751003, Odisha, India
}

\section{ABSTRACT}

Introduction: Choanal atresia $(\mathrm{CA})$ is the commonest congenital anomaly of the nose. The diagnosis is made by diagnostic nasal endoscopy and computed tomography (CT) scan. The definitive treatment option is surgery.

Aim of the study: To discuss our experiences with CA in our region and factors affecting the outcome. Material and methods: A prospective study of paediatric patients done between 2012 and 2017. All patients were evaluated by diagnostic nasal endoscopy and CT of the nose and paranasal sinuses. All were operated by endonasal endoscopic approach with placement of a stent. Patients were followed-up for an average of one to two years.

Results: All 21 patients of CA were treated with endoscopic trans-nasal technique. No severe postoperative complications were found except three cases resulting in restenosis. The restenosis cases underwent revision surgery.

Conclusions: The endonasal endoscopic approach is a safe and effective method for the choanal atresia. Placement of a stent prevents restenosis with good outcome.

\section{KEY WORDS:}

choanal atresia, endoscopic endonasal surgery, nasal stent, congenital malformation.

\section{INTRODUCTION}

Choanal atresia (CA) is defined as an obliteration of posterior choanal aperture and was first documented by Roderer in 1775, whereas Otto described it in better way, considering its anatomical anomaly [1]. The average rate of CA is 0.82 cases per 100,000 individuals. Unilateral CA occurs more commonly in the right nasal cavity, and the ratio of unilateral to bilateral cases is 2:1 [2]. The congenital CA occurs due to failure of the bucconasal membrane to rupture, persistence of the buccopharyngeal membrane, medial outgrowth of the vertical and horizontal process of the palatine bone, and probable defects in the neural crest migration [3]. The anatomical deformity leading to CA are lateral bony obstruction by the lateral pterygoid plate, medial obstruction by the thickened vomer, narrow nasal cavity, and a membranous obstruction [4]. There are three types of CA: bony, membranous, and mixed (bony-membranous atresia) [5]. The majority of the patients (70\%) have mixed type of CA [6]. CA is seen unilaterally $(60 \%)$ and bilaterally in $40 \%$ of cases. Unilateral CA may not be detected in the first few years of life and usually presents with unilateral rhinorrhoea or nasal obstruction [7]. 20-50\% of CA patients particu-

\section{ADDRESS FOR CORRESPONDENCE:}

Dr. Santosh Kumar Swain, Prof., Department of Otorhinolaryngology, IMS and SUM Hospital, Bhubaneswar,

Odisha, India, phone: 09556524 887, e-mail: santoshvoltaire@yahoo.co.in 
larly bilateral one associated with other malformations like CHARGE (Coloboma, heart defects, choanal atresia, retardation of postnatal growth and mental development, genital hypoplasia, and ear anomalies), Treacher Collins, Crouzon, Pfeiffer, Apert, and mandibulofacial dysostosis syndrome [8]. Failure of the bucconasal membrane of Hochstetter to rupture results in CA. Several aetiological factors have been hypothesised. Maternal hyperthyroidism treated with anti-thyroid drugs like methimazole or carbimazole are implicated as causing choanal atresia [9]. In this study, we discuss the clinical profile, management, and outcomes of paediatric CA presenting to our centre.

\section{MATERIAL AND METHODS}

A prospective study was conducted in the department of otorhinolaryngology of a tertiary care teaching hospital in eastern India between December 2012 and July 2017. Patients coming directly to the otolaryngology depart-

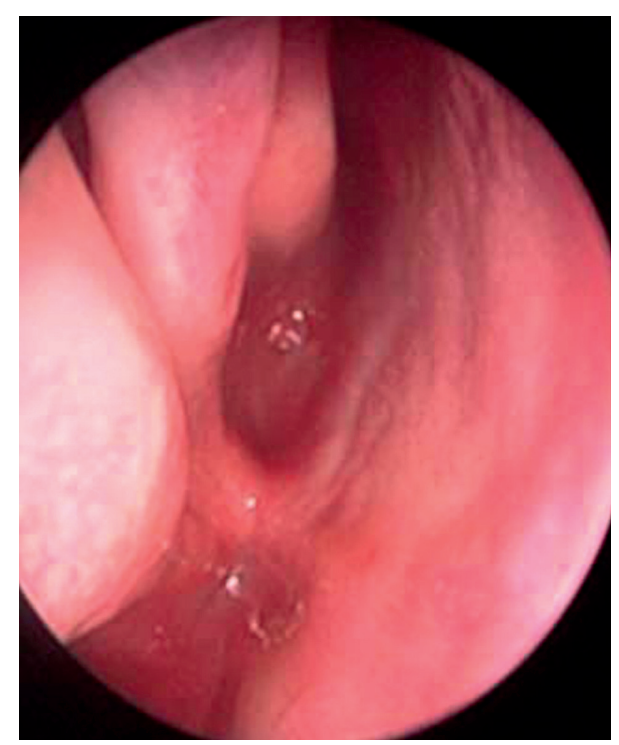

FIGURE 1. Endoscopic picture of choanal atresia

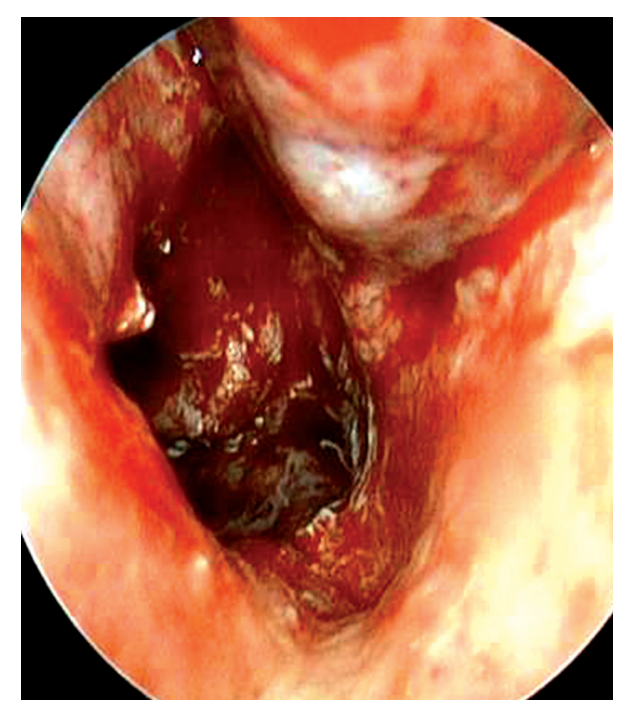

FIGURE 3. Neo-choana made after widening by endoscopic approach ment or referred from the paediatric department were included in the study. Complete clinical examinations were done to assess any other congenital anomalies. Initially a small feeding tube was used to assess the patency of the choana and then a flexible fibreoptic endoscope was used for confirmation of the deformity. After endoscopic evaluation (Fig. 1), imaging was done with non-contrast CT scan of the nose and paranasal sinuses (Fig. 2). Management of CA was done depending on the age of the patient, type of CA, and laterality (unilateral/bilateral). All paediatric patients were treated with trans-nasal endoscopic approach. In bony choanal atresia, posterior septectomy was done and the bony part excised along the most inferomedial aspect and widened appropriately (Fig. 3). In mixed choanal atresia, the membranous part was perforated by suction tip and the bony part was removed by curettes, Kerrisons punch, or drilling with a burr. Stenting was done after widening the choana with an appropriate feeding tube or endotracheal tube (Fig. 4).

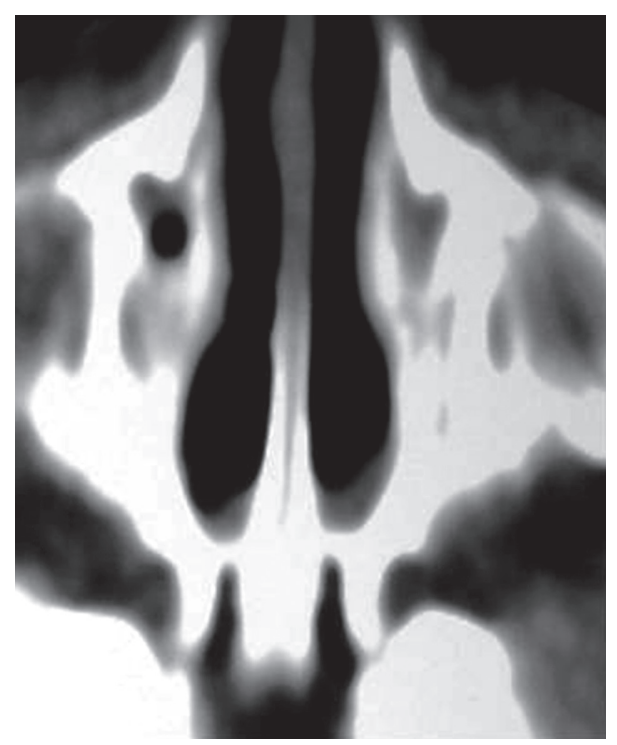

FIGURE 2. CT scans (axial cut) showing bilateral choanal atresia

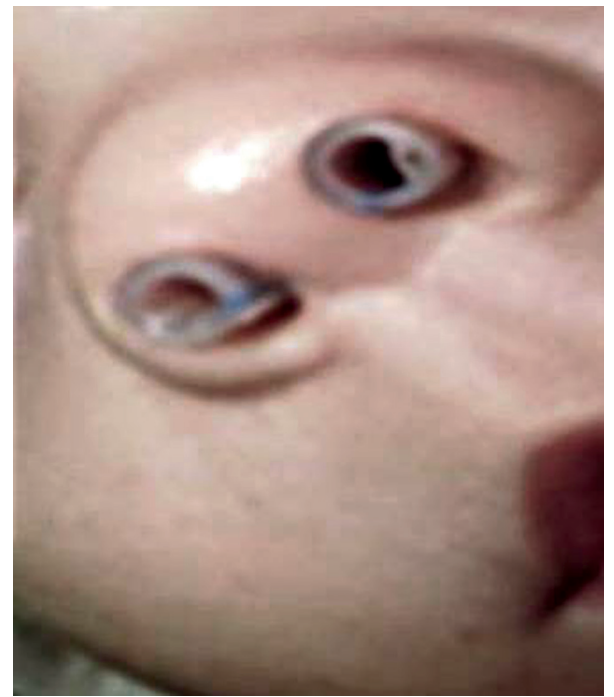

FIGURE 4. Placement of stent in bilateral neochoana 
The stent was removed after four weeks. All children were kept with anti-reflux management for four weeks to prevent granulation formation. Patients were discharged from the hospital 2 to 14 days after surgery, depending on the associated problems and where the patients lived. After removal of stents, patients were regularly followedup with nasal endoscopy at the outpatient department of otolaryngology for one to two years.

\section{RESULTS}

There were 21 cases of CA in this study with $12 \mathrm{fe}$ males and nine males. Sixteen patients $(76.19 \%)$ had unilateral CA and five (23.80\%) had bilateral CA. Seven (33.33\%) had pure bony CA and fourteen $(66.66 \%)$ had mixed bony membranous CA (Table 1 ). All the children with bilateral CA were operated within 15 days of birth. The patients with unilateral CA had an age range of six to 13 years with an average of 9.8 years. All the children underwent trans-nasal endoscopic surgery and the neochoana was covered with mucoperiosteum with placement of an appropriately sized stent (made from feeding or endotracheal tube). There were no major intra-operative or postoperative complications except for three cases that developed restenosis at choana (Table 2). Out of the 21 children, three patients needed revision surgery of drilling later on for persistent bony stenosis. Patients with bilateral CA needed airway stabilisation before surgery by endotracheal intubation in two cases $(9.52 \%)$, oral cannula in two cases $(9.52 \%)$, and McGovern nipple in one case (4.76\%). One patient was suffering from CHARGE syndrome.

\section{DISCUSSION}

Choanal atresia is a clinical entity characterised by obliteration of the posterior nasal aperture causing upper airway obstruction, which may be congenital or acquired in origin. CA is the commonest congenital anomaly of the nose, and the global incidence of CA documented in the medical literature is one per 8000-10,000 live births [10]. It is more commonly seen in females, and the most common type of atretic plate is right unilateral [11]. The mixed type (consisting of bony and membranous components) accounts for $70 \%$ while the bony type accounts for $30 \%$ of cases [12]. In our series, 16 patients (76.19\%) had unilateral CA, five (23.80\%) had bilateral CA, seven (33.33\%) had pure bony CA, and 14 (66.66\%) had mixed bony membranous CA. Acquired CA is extremely rare and often caused by rhinopharyngeal injury, e.g. after adenoidectomy, radiation effects after nasopharyngeal carcinoma, syphilis or tuberculosis of the nasopharynx, and sometimes the aetiology is unknown [13]. In the case of bilateral congenital CA, some authors found its association with low thyroxin levels in infants [14]. Kancheral et al. documented evidence linking CA to maternal exposure to different nutrients, thyroid medications, and
TABLE 1. Patients's profile

\begin{tabular}{|l|c|}
\hline Number of patients & 21 \\
\hline Gender & 12 \\
Male & 9 \\
Female & \\
\hline Age of the patients & 3 days to 11 years \\
Range & 6.5 years \\
Mean((SD)-Unilateral & 5.6 days \\
Mean(SD)-Bilateral & \\
\hline Side & 16 \\
Unilateral & 5 \\
Bilateral & \\
\hline Type of choanal atresia & 14 \\
Mixed & 7 \\
Bony & \\
\hline
\end{tabular}

TABLE 2. Postoperative complications in choanal atresia

\begin{tabular}{|l|c|}
\hline Complications & Number of patients \\
\hline Restenosis at the choana & 3 \\
\hline Synechiae in the nasal cavity & 2 \\
\hline Epistaxis & 1 \\
\hline Accidental extraction of stent & 1 \\
\hline Respiratory obstruction due to stent block & 1 \\
\hline Necrosis of the nasal mucosa & 1 \\
\hline
\end{tabular}

cigarette smoking [15]. There is an association between pre-pregnancy exposure and CA are intake in the highest quartile: vitamin $B_{12}$, zinc, niacin; intake in the lowest quartile: methionine, vitamin-D; coffee (more than three cups/day). Symptoms like severe airway obstruction and cyclical cyanosis are the classical signs for neonatal bilateral CA. When babies cry, they alleviate respiratory distress because they are obligate nasal breathers, and so the neonatologist should be alerted for the probability of bilateral CA. These were the classical features evidenced in our bilateral CA. Patients with unilateral CA may be suspected at birth by a snorting cry and the inability to pass a suction catheter, although clinical presentation is often delayed with symptoms of nasal obstruction, nasal discharge, delayed speech, and mouth breathing. Bilateral CA causes respiratory distress in the neonate and the patient presents with cyclic cyanotic spells that improve with crying. Bilateral CA needs immediate intervention, unless the airway is supported with an oral airway. Delayed treatment is occasionally sought to allow nasal and facial growth. Unilateral CA may not be diagnosed for years, and patients usually present with unilateral nasal obstruction or rhinorrhoea. In our series, the majority of the patients were unilateral CA. The differential diagnosis of CA includes stenosis of the pyriform aperture, and nasal mass like glioma, encephalocele, dermoid cysts, septal deviation, and foreign bodies [16]. Patients should be properly examined to find and treat the possible as- 
sociated anomalies or malformations (seen in $20 \%$ to 50\%) such as CHARGE [8]. Bilateral and complete CA is a medical emergency, which requires an urgent airway, whereas patients with incomplete or unilateral CA are often diagnosed and treated in the later part of life when the patient complains of long-standing unilateral nasal obstruction, rhinorrhoea, or anosmia. Children with CA suffer from feeding difficulties, failure to thrive, oxygen desaturation, and cyanosis. The clinical evaluation of CA is done with a flexible fibreoptic endoscope. CT scan is the imaging method of the choice for evaluation of CA [17]. In the acute phase of CA, the management includes oropharyngeal airway, like a Guedel airway or endotracheal intubation, but surgery is the definitive treatment. The treatment of CA is as important as its diagnosis. Bilateral CA needs emergency airway maintenance. Oral airway between the palate and tongue can be facilitated for this purpose before the definitive treatment. Once the diagnosis of CA is confirmed, surgery is the only treatment option. There are several surgical approaches, e.g. trans-nasal, trans-palatine, trans-antral, and trans-septal. The objective of the surgery in the CA is to remove the atretic plate completely and assure long-term patency. This surgery needs detailed preoperative assessment and good intra-operative visualisation [18]. The trans-palatine approach provides a wide field for surgery but is more susceptible to complications like bleeding, fistulas, infections, defects in jaw growth, and palatine bone [19]. The trans-antral approach has only historical interest, but it also gives good exposure of the surgical filed with significant risk of deformities of growing facial bones. The trans-septal approach is advised in cases of unilateral CA in older children. The trans-nasal approach is nowadays the most commonly used due to the modern refinement of endoscopic techniques. It does not affect the growth of the mandibular arch, so there are no future malocclusions or cosmetic problems. The trans-nasal endoscopic approach is very useful in newborns with bilateral CA, where a puncture is made on the atretic bony plate and widened with Fearon dilators and stent placement [20]. The endoscopic endonasal approach for the treatment of CA is a safe and effective method for both unilateral and bilateral CA. The accurate removal of the posterior edge of the vomer and inferior part of the sphenoid rostrum along with complete covering of the bony margins of the neochoana by mucoperiosteal flaps are important steps to prevent postoperative restenosis. Recently, balloon dilatation has been used widely as a surgical method in otolaryngology and is established particularly in endoscopic sinus surgery [21]. It is successful already in subglottic and tracheal stenosis. The advantage of balloon dilators is the application of a radial force, which avoids the shearing forces associated with other dilating methods [22]. It has another advantage: the catheter has a very small diameter, so the balloon can be easily inserted through the nasal cavity of the neonates without damage to the adjacent tis- sue. Post-operative formation of synechia, scar tissue, and granulation are usually reported and may lead to recurrent nasal obstruction. Adjuvant mitomycin and stenting have been used over the years to reduce these problems. Postoperative restenosis occurs due to excess granulation and scar tissue in the operative field (concentric wound surface). Therefore, the intra-operative tissue injury has to be minimised as much as possible. Some authors have tried application of mitomycin C on the neochoana to improve healing, reduce scarring, and lower the incidence of restenosis [23]. However, many authors have reported no convincing effect on long-term success [24]. Mitomycin C is a topical aminoglycoside antibiotic reducing scar and granulation formation by inhibiting fibroblast growth and migration [25]. In our study, placement of a stent at the neochoana was done without application of mitomycin C. Advanced technology like LASER and microdebrider now provide more effective tools for managing CA. The trans-nasal endoscopic technique with or without powered instrumentation provides excellent exposure with great ease in removing the atretic plate of CA [26].

\section{CONCLUSIONS}

Congenital choanal atresia is an uncommon clinical entity. Use of endonasal endoscopic approach for the treatment of CA dramatically changed the outcome. The accurate removal of the posterior edge of the vomer and inferior portion of the sphenoid rostrum as well as the covering of the neochoana by mucoperiosteum flaps are key techniques for preventing stenosis. The use of a stent is an additional technique for avoiding postoperative restenosis. Further studies are needed for detailed evaluation of CA and its treatment outcome. However, due to the low number of patients included in this study, a larger sample is needed to draw statistically significant conclusions.

\section{DISCLOSURE}

The authors declare no conflict of interest.

\section{REFERENCES}

1. Pasquini E, Sciarretta V, Saggese D, et al. Endoscopic treatment of congenital choanal atresia. Int J of Pediatr Otorhinolaryngol 2003; 67: 271-276.

2. Freitas RP, Berkowitz RG. Bilateral choanal atresia repair in neonates: a single sugeon experience. Int J Pediatr Otorhinolaryngol 2012; 76: 873-878.

3. Fulton RC, Banerjee J, Chow P, Godambe SV. Preoperative airway management in a preterm with bilateral choanal atresia. J Perinatol 2000; 27: 521-522.

4. Keller JL, Kacker AMD. Choanal atresia, charge association and congenital nasal stenosis. Otolaryngol Clin North Am 2000; 33: 1343-1351.

5. Durmaz A, Tosun F, Yldrm N, et al. Transnasal endoscopic repair of choanal atresia: results of 13 cases and meta-analysis. J Craniofac Surg 2008; 19: 1270-1274. 
6. Wootten CT, Elluru RE. Congenital malformations of the nose. In: Cummings Otolaryngology Head \& Neck Surgery. vol. 3. Flint PW (ed.). $5^{\text {th }}$ ed. Mosby Elsevier, Philadelphia 2010; 2686-2696.

7. Riepl R, Scheithauer M, Hoffmann TK, Rotter N. Transnasal endoscopic treatment of bilateral choanal atresia in newborns using balloon dilatation: own results and review of literature. Int $J$ Pediatr Otorhinolaryngol 2014; 78: 459-464.

8. Velegrakis S, Mantsopoulos K, Iro H, Zenk J. Long-term outcomesof endonasal surgery for choanal atresia: 28 years experience in an academic medical centre. Eur Arch Otorhinolaryngol 2013; 270: 113-116.

9. Barbero P, Valdez R, Rodriguez H, et al. Choanal atresia associated with maternal hypothyroidism treated with methimazole: a case-control study. Am J Med Genet A 2008; 146A: 2390-2395.

10. Lee LJ, Canfield MA, Hashmi SS, et al. Association between thyroxine levels at birth and choanal atresia or stenosis among infants in Texas, 2004-2007. Birth Defects Res A Clin Mol Terato 2012; 94: 951-954.

11. Newman JR, Harmon P, Shirley WP, et al. Operative management of choanal atresia: a 15-yearexperience. JAMA Otolaryngol Head Neck Surg 2013; 139: 71-75.

12. Corrales CE, Koltai PJ. Choanal atresia: current conceptsand controversies. Curr Opin Otolaryngol Head Neck Surg 2009; 17: 466470.

13. Assanasen P, Metheetrairut C. Choanal atresia. J Med Assoc Thai 2009; 92: 699-706.

14. Chia SH, Carvalho DS, Jaffe DM, Pransky SM. Unilateral choanal atresia in identical twins: case report and literature review. Int J Pediatr Otorhinolaryngol 2002; 62: 249-252.

15. Kancherla V, Romitti PA, Sun L, et al. Descriptive and risk factor analysis for choanal atresia: The national Birth defects prevention Study, 1997-2007. Eur J Med Genet 2014; 57: 220-229.

16. Zanetta A, Rodríguez H, Quiroga V, et al. Congenital nasal obstruction due to choanal atresia. Arch Argent Pediatr 2012; 110: 152-155.

17. Chaudhary V, Anand R, Narula MK, Singh SK. The role of computed tomography in the evaluation of bilateral choanal atresia: two case reports. Iran J Radiol 2009; 6: 221-224.

18. Shawky ME. Transnasal endoscopic management of congenital bilateral choanal atresia with and without stenting. PAN Arab J Rhinol 2011; 1: 17-22.

19. Kwong KM. Current updates on choanal atresia. Front Pediatr 2015; 3: $1-6$.

20. Gujrathi CS, Daniel SJ, James AL, Forte V. Management of bilateral choanal atresia in the neonate: an institutional review. Int J Pediatr Otorhinolaryngol 2004; 68: 399-407.

21. Ramsden JD, Campisi P, Forte V. Choanal atresia and choanal stenosis. Otolaryngol Clin North Am 2009; 42: 339-352.

22. Bedwell J, Shah RK, Baumann N, et al. Balloon dilation for management of choanal atresia and stenosis. Int J Pediatr Otorhinolaryngol 2011; 75: 1515-1518.

23. Prasad M, Ward RF, April MM, et al. Topical mitomycin as an adjunct to choanal atresia repair. Arch Otolaryngol Head Neck Surg 2002; 128: 398-400,

24. Newman JR, Harmon P, Shirley WP, et al. Operative management of choanal atresia: a 15-year experience. JAMA Otolaryngol Head Neck 2013; 139: 71-75.

25. Carter JM, Lawlor C, Guarisco JL. The efficacy of mitomycin and stenting in choanal atresia repair: A 20 year experience. Int J Pediatr Otorhinolaryngol 2014; 78: 307-311.

26. Assanasen P, Metheetrairut C. Choanal atresia. J Med Assoc Thai 2009; 92: 699-706. 